\title{
Arterial stiffness in patients with non-classic Pompe disease: role of antihypertensive drugs and statins
}

\author{
Ercan Varol
}

Received: 21 March 2014 / Accepted: 17 April 2014 / Published online: 10 May 2014

(C) SSIEM and Springer Science+Business Media Dordrecht 2014

I read the article "Increased aortic stiffness and blood pressure in non-classic Pompe disease" by Wens et al. with great interest (Wens et al. 2014). They investigated the arterial stiffness in patients with Pompe disease. They found that patients with Pompe disease had significantly higher pulse wave velocity values as compared to controls. This difference was still present after adjustment for age, gender, mean arterial blood pressure, heart rate and diabetes mellitus.

They concluded that patients with non-classic Pompe disease have increased aortic stiffness and blood pressure. I congratulate the authors for this important study. However, I want to make minor criticism for this study from the methodological aspect.

It has to be kept in mind that arterial stiffness is a complex process. It has been previously thought that it is closely associated with atherosclerotic risk factors such as smoking, alcohol consumption, hypercholesterolemia, hypertension and older age. Recently, Cecelja et al. published a systematic review which showed that the contribution of cardiovascular risk factors other than age and blood pressure to aortic stiffness measured by carotid-femoral pulse wave velocity is small or insignificant and age and blood pressure were consistently independently associated with aortic stiffness (Cecelja and Chowienczyk 2009). As a result, this systematic review showed that age and blood pressure were consistently independently associated with aortic stiffness in the majority of studies. It has also been shown that some antihypertensive drugs like angiotensin-converting enzyme inhibitors, calcium channel blockers and spiranolactone reduce arterial stiffness (Cavalcante et al. 2011; Dudenbostel and Glasser 2012). On the other hand, the role of angiotensin-2 receptor blockers on arterial stiffness is not yet clear. Also statins reduce arterial

Communicated by: Maurizio Scarpa

E. Varol $(\bowtie)$

Tip Fakultesi, Suleyman Demirel Univesitesi, Isparta, Turkey

e-mail: drercanvarol@yahoo.com stiffness (Cavalcante et al. 2011). When we look at the study, there are hypertensive and hyperlipidemic patients and controls. However, there is no data of antihypertensive drugs used in detail. From this aspect, which antihypertensive drug is used is important. There is also no data of statin use in hypercholesterolemic patients and controls. These factors can influence the results. It would have been useful if the authors had provided this information.

Arterial stiffness describes the reduced capability of an artery to expand and contract in response to pressure changes and it is an independent predictor of cardiovascular morbidity and all-cause mortality (Cavalcante et al. 2011). It has been suggested that aortic stiffness occurs as a result of atherosclerosis along the aorta. However, it is closely associated with age and hypertension and antihypertensive drugs. Antihypertensive drugs and statins can greatly influence arterial stiffness and these factors should be considered in arterial stiffness evaluation.

Conflict of interest None.

Informed consent This article does not contain any studies with human or animal subjects performed by the any of the authors.

\section{References}

Cavalcante JL, Lima JA, Redheuil A, Al-Mallah MH (2011) Aortic stiffness: current understanding and future directions. J Am Coll Cardiol 57(14):1511-1522

Cecelja M, Chowienczyk P (2009) Dissociation of aortic pulse wave velocity with risk factors for cardiovascular disease other than hypertension: a systematic review. Hypertension 54(6):1328-1336

Dudenbostel T, Glasser SP (2012) Effects of antihypertensive drugs on arterial stiffness. Cardiol Rev 20(5):259-263

Wens SC, Kuperus E, Mattace-Raso FU et al. (2014) Increased aortic stiffness and blood pressure in non-classic Pompe disease. J Inherit Metab Dis. doi:10.1007/s10545-013-9667-2 